\title{
Miasi congiuntivale da Oestrus ovis: descrizione di un nuovo caso e problematiche in tema di miasi in ambito umano
}

\author{
Maria Letizia D’Annibale', Daniele Crotti² \\ 'Struttura Complessa di Microbiologia, Azienda Ospedaliera di Perugia, Perugia \\ ${ }^{2}$ L. P. \& L. D. in Parassitologia e Microbiologia Medica, Perugia
}

Key words: myasis, Oestrus ovis, ophtalmomyasis

Ophtalmomyasis due to Oestrus ovis: a case report.

\section{SUMMARY}

The Authors related a third case of ophtalmomyasis occurred to a young adult female, during holidays in Sardinia, near a beed of reeds bordering on the beach in the north of the island.Various larvae were captured in the eyeconjunctiva and three were identified at the optical microscope as first stadium larvae of Oestrus ovis on the base of typical and reported features and characters. The Authors relate the main diagnostics keys and algorithmes for the myasis that could affect humans, as in Italy as in developing countries, and suggest a major attention to these renewed clinical pathologies caused by larvae of various genera and species of flies, not only in natural cavities (like conjunctiva) but also on the cutaneous and subcutaneous tissues, and in intestinal tract.

\section{INTRODUZIONE}

Le miasi (dal termine greco muia: mosca) sono parassitosi causate da larve di mosche, nella fattispecie da ditteri ciclorrafi appartenenti a diverse famiglie, generi e specie (19).

Da un punto di vista epidemiologico le miasi vengono classificate in miasi accidentali, miasi facoltative e miasi obbligatorie (12)

Da un punto di vista clinico (e di localizzazione d'organo e/o tessuto) possono venire classificate in miasi cutanee (o superficiali), miasi foruncolose (o sottocutanee), miasi intestinali, miasi cavitarie (tra cui le forme congiuntivali) e miasi profonde o con migrazione interna $(12,19)$. Tutte queste miasi rientrano nelle forme facoltative o profonde (vedi oltre).

Le miasi accidentali sono quelle provocate da larve ingerite con alimenti avariati o lavati con acque contaminate. La loro ingestione, peraltro, può essere involontaria (miasi accidentali vere $\mathrm{e}$ proprie) o volontaria. Nel primo caso sono coinvolte Musca domestica e Sarcophaga cartaria (25); nel secondo caso essenzialmente Piophila casei, il "verme del formaggio $(12,19,20)$.

Clinicamente possono essere responsabili di disturbi gastro-intestinali, anche violenti, ma a prognosi sempre benigna $(12,20)$.

Le miasi facoltative sono causate da ditteri che normalmente si nutrono su corpi di animali morti o su materiale organico in decomposizione (nell'uomo su lesioni e ferite purulente o necrotiche) e sul quale facoltativamente possono essere depositate uova o lanciate direttamente le larve (talora anche in cavità naturali, quali, ad esempio, il condotto uditivo esterno o la vagina). Sono coinvolte le seguenti mosche: Lucilia spp. (la "mosca verde"), Phormia spp. (la "mosca nera", europea e nordamericana), Calliphora spp. (la "mosca azzurra"), Chrysomyia spp. (solo in Africa, Asia e Australia), Cochlyomyia spp. (americana), Auchmeromyia spp. (in Africa sub-sahariana), Sarcophaga spp., e Wohlfahrtia spp., quest'ultima presente solo in Europa (9, 11, 12, 19, 20). Le prime sei sono ovipare, mentre le ultime due sono larvipare (19). Sono tutte responsabili di miasi cutanee o superficiali, più raramente con coinvolgimento del tessuto sottocutaneo $(9,12)$.

Le miasi obbligatorie comprendono specie parassite obbligatorie che si localizzano allo stadio larvale in tessuti vivi ove compiono una parte del ciclo vitale; le più comuni vie di penetrazione sono le ferite, gli orifizi corporei esterni, la cute anche integra $(12,20)$. Tra le miasi obbligatorie annoveriamo le miasi foruncolose (o, come detto, sottocutanee), le miasi cavitarie, le miasi intestinali vere e proprie, le miasi profonde $(12,20)$.

Nelle miasi foruncolose le larve, penetrate attraverso la cute, si sviluppano nello spessore del derma e del sottocutaneo provocando la formazione di un granuloma reattivo (19). Vi appartengono Cordylobia spp. (mosca africana) e Dermatobia spp. (mosca americana). 
Nelle miasi cavitarie le larve vengono "lanciate" all'interno di cavità corporee naturali quali fosse nasali, fauci, orecchio esterno e fornici congiuntivali degli occhi (da cui, appunto, le miasi congiuntivali). Ne sono responsabili Oestrus ovis e, più raramente, Rhinoestrus spp. $(12,19)$.

Nelle miasi intestinali le larve, introdottesi per via orale, passano parte del loro ciclo in tratti del tubo digerente, causando miasi gastrica o miasi rettale; queste sono causate da specie del genere Gasterophilus $(12,19)$.

Infine, nelle miasi cosiddette profonde, le larve, penetrate per via orale o attraverso la cute, devono compiere migrazioni obbligatorie all'interno dell'ospite prima di emergere a livello cutaneo. Appartengono tutte al genere Hypoderma, ma l'uomo ne è colpito assai raramente $(12,19)$.

Nell'uomo, attualmente, sono descritte soprattutto le forme foruncolose causate da Dermatobia $(5,18)$ o Cordylobia $(14,24)$, in soggetti rientranti rispettivamente da regioni centro-sudamericane o africane, e le forme cavitarie, in particolare quelle congiuntivali (o oculari) causate da Oestrus ovis $(2,3,4,7,15)$.

\section{CASO CLINICO}

Una giovane adulta italiana è, a fine luglio, in vacanza in Sardegna, a Santa Teresa di Gallura. È sulla spiaggia; dietro, in fondo alla spiaggia, vi è un canneto (dirà un amico successivamente). Improvvisamente vede una piccola massa scura vicino all'occhio e quindi un leggero dolore nello stesso. Comincia a sentire fastidio e bruciore. Il giorno appresso rientra a Perugia con amici del posto, ma soltanto di passaggio in quanto la stessa vive in provincia di Milano. Durante il viaggio di ritorno sopporta il dolore, ma non appena arrivata a Perugia si presenta al PS del capoluogo umbro dove le vengono estratte alcune larve (riferite come "larve o uova o insetti" con dei punti neri) e le consigliano collirio antinfiammatorio. Persiste il disturbo, per cui il giorno successivo torna dall'oculista che, dopo un attento esame, osserva come dei "piccoli insetti" mobili; lo stesso telefona al laboratorio parassitologico chiedendo se può inviare tali elementi per avere un conforto diagnostico di certezza. Vengono così presi accordi sulle modalità di raccolta e di invio di tali sospette larve. L'oculista estrae svariate larve (non viene però precisato quante) e tre vengono inviate al laboratorio parassitologico attraverso la paziente stessa (che a sua volta consegnerà il referto all'oculista nel corso della medesima giornata); qui vengono subito individuate come larve di I stadio di Oestrus ovis (Figure I, II, III).

Le larve sono biancastre, mobili, di lunghezza di poco superiore ad $1 \mathrm{~mm}$.
Le caratteristiche diagnostiche morfologiche microscopiche relative ad $O$. ovis, oltre alla sede di reperimento, sono rappresentate da:

1) segmento (o anello) cefalico con 2 uncini buccali a forma di "corna di toro" (Figura I);

2) le spine dei segmenti del corpo (più di 11; Figura II e Figura III);

3) gli spiracoli respiratori del segmento terminale (nel nostro caso non evidenziabili).

\section{Caratteristiche diagnostiche differenziale delle larve coinvolte nelle miasi}

Come accennato, la diagnostica è morfologica essenzialmente microscopica. Essa si basa:

1) sulla morfologia, numero e caratteristiche dei segmenti del corpo;

2) sulla tipologia del segmento cefalico;

3) sulle particolarità degli spiracoli dell'ultimo segmento o caudale, che sono tipici per ogni specie di larva di mosca responsabile di una miasi (4).

Mentre però le prime due caratteristiche morfologiche delle larve si possono osservare con l'utiliz-

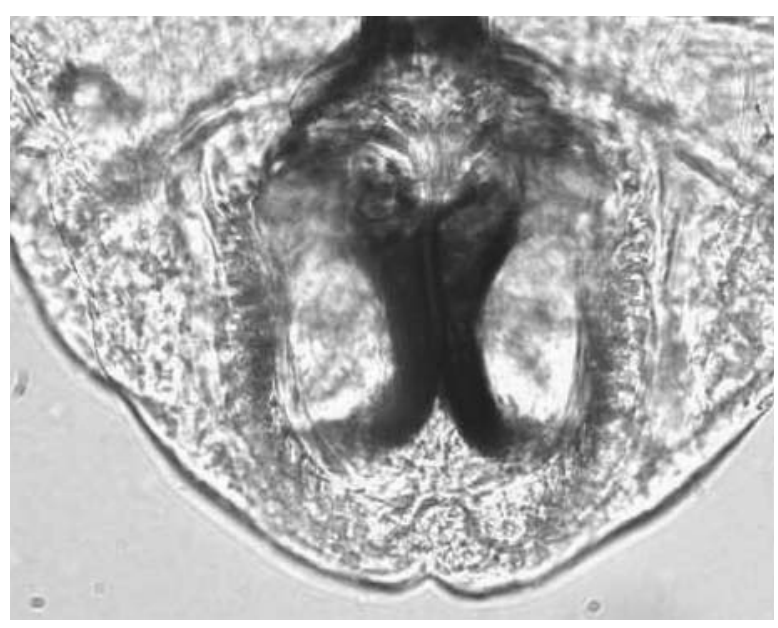

Figura I. Larva I di I stadio di Oestrus ovis: particolarità del segmento cefalico (gli uncini boccali a forma di corna)

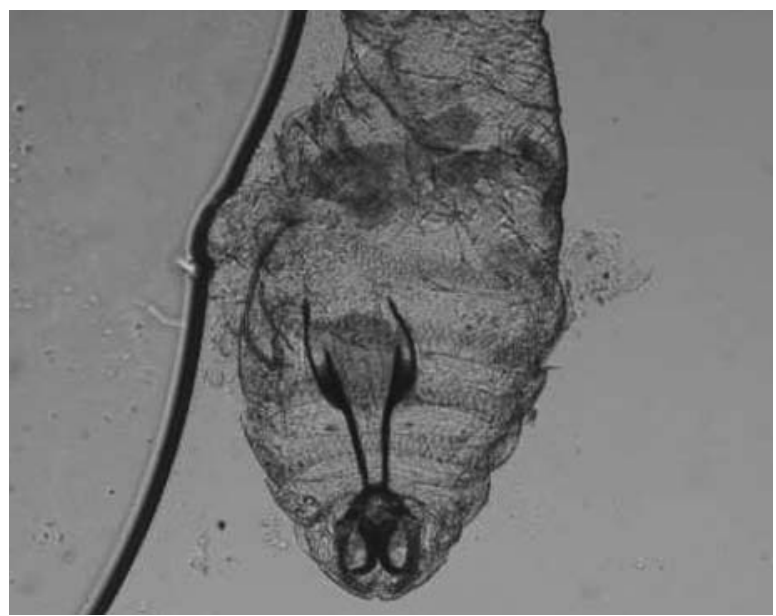

Figura II Larva di I stadio di O. ovis: segmento cefalico con uncini e le spine dei primi segmenti del corpo 


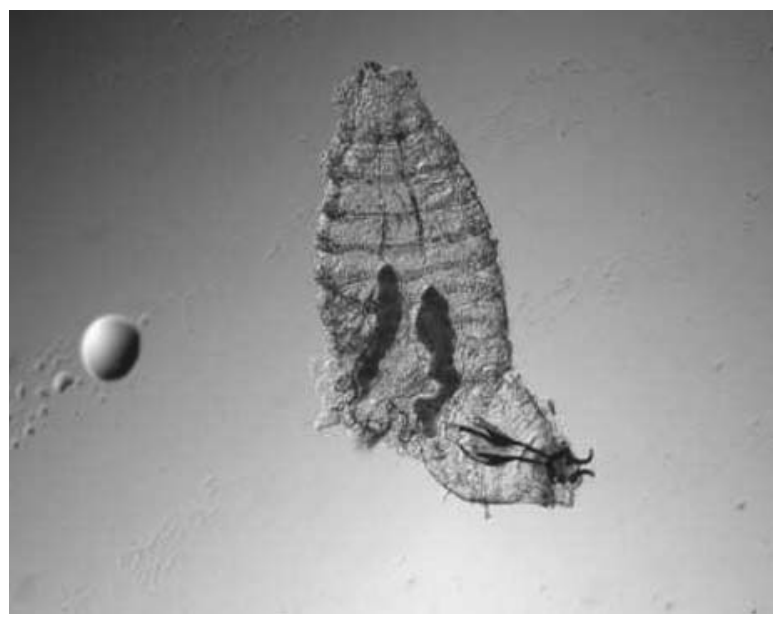

Figura III. Larva di I stadio di O. ovis (completa)

zo del microscopio ottico, le peculiarità degli spiracoli è apprezzabile di fatto soltanto istologicamente, dopo colorazioni specifiche (6).

\section{DISCUSSIONE}

Abbiamo voluto ricordare le principali problematiche relative a tutte le miasi umane, in quanto sono parassitosi recentemente tornate alla ribalta clinica $(2,3,4,7,15)$ dopo anni di apparente dimenticanza $(16,17)$, forse per riemersa attenzione a tali zoonosi (17).

Se è vero che spesso, nelle forme cutanee e sottocutanee si è costretti ad eseguire una diagnostica istologica, in quanto inavvertitamente o troppo tempestivamente vengono eseguite biopsie su noduli, granulomi o tumefazioni cutanee $(7,11)$, è altresì vero che laddove raccolte, estratte o prelevate intatte, per la diagnostica di tali larve è necessario conoscere o comunque ricordarsi dei principali caratteri morfologici, utili e necessari alla loro identificazione $(2,5)$.

In particolare in tema di miasi cavitarie e, nella fattispecie, di miasi congiuntivali (od oftalmomiasi), forse da noi le più frequenti anche perché autoctone, è importante conoscere le caratteristiche morfologiche, o potere consultare adeguati testi per riconoscerle, sì da avere la certezza di una corretta identificazione, sebbene la sede di estrazione della larva stessa possa essere già orientativa nell'inquadramento eziologico, ovvero sia nella diagnosi identificativa $(2,5)$.

Le miasi congiuntivali (od oculari) sono ben documentate in campo veterinario, sia nel Vecchio come nel Nuovo Mondo, vuoi da un punto di vista epidemiologico, vuoi da un punto di vista diagnostico $(1,22)$. In Europa, sempre in campo animale, importanti sono gli attualissimi studi in ambito immunologico e preventivo-profilattico riguardanti ovviamente la popolazione ovina $(21,23)$.

In campo umano le segnalazioni, in Italia, si sono recentemente incrementate $(4,6,7)$ e ben si possono confrontare con quanto riportato in questi ultimissimi anni anche in altre regioni dell'area mediterranea o a questa strettamente vicina ed in parte affine $(8,10,13)$.

Concludiamo ricordando come, in campo parassitologico ed in particolare entomologico una stretta collaborazione tra medici e veterinari non può che essere importante ed indubbiamente foriera di maggiore conoscenza ed attenzione, nell'ambito specificatamente umano, per patologie spesso misconosciute o trascurate $(14,16)$.

\section{BIBLIOGRAFIA}

1. Alcaide M, Reina D, Frontiera E, Navarrete I, Analysis of larval antigens of Oestrus ovis for the diagnosis of oestrosis by enzyme-linked immunosorbent assay. Med. Vet. Entomol. 2005; 19: 151-157.

2. Cianchetti A, Ricci AL, Antei R, Piergili D (1987). Su di un caso di oftalmomiasi accorso nella regione umbra. Ann. Ottalm. Cl. Ocul. 1987; Vol. CXIII: $157-$ 163

3. Crotti D, Cianchetti A. Un caso di miasi congiuntivale umana da Oestrus ovis. GIMMOC 2004; Vol. III: 186-189.

4. Crotti D, D'annibale ML, Ricci A. Miasi congiuntivele umana da Oestrus ovis: descrizione di un caso, considerazioni generali e indicazioni diagnostico-epidemiologiche. Le Infezioni in Medicina 2005; 2: 120122.

5. Crotti D, Prosperini AP. Miasi foruncolosi di importazione: descrizione di un caso. G. It. Med. Trop. 2004; 9: 95-97.

6. Dono M, Bertonati MR, Poggi R, et al. Three cases of ophthalmomyiasis externa by sheep botfly Oestrus ovis in Italy. XXXV Congresso Nazionale AMCLI, Torino, 19-22 settembre, 2006, Poster $\mathrm{N}^{\circ}$ 68, Microbiol. Med. 2006; 21: 212.

7. Fazii P, Cosentino L, Carusi T, et al. Miasi umane causate da Oestrus ovis (Diptera: Ostridae) con descrizione di un caso di oftalmomiasi esterna bilaterale. GIMMOC 2006; Vol. X: 21-25.

8. Fathy FM, El-Barghati A, El-Ahwal A, El-Bagar S. Study of human ophthalmomyiasis externa caused by Oestrus ovis in Libya: parasite features, clinical presentation and management. J. Egypt. Soc. Parasitol. 2006; 36: 265-282.

9. Garcia LS. Diagnostic Medical Parasitology. ASM Press, Washington D.C., 2001, IV Edition, 658-665.

10. Hakimi R, Yazdi I. Oral mucosa myasis caused by Oestrus Htpp://www.ams.ac.ir/AIM/0253/0253194.htm.

11. Iori A, Zechini B, Cordier L, et al. A case of human myasis of the scalp due to Wohlfahrtia magnifica recorded near Rome. Parassitologia 1998; 40 (Suppl. 1): 82 .

12. Manfredini L. Insetti e acari di importanza veterinaria e medica. Mazzanti Editore, Venezia, 2005, 42-47.

13. Masoodi M, Hosseini K. External ophthalmomyiasis caused by sheep botfly (Oestrus ovis) larva: a report of 8 cases. Arch. Iranian Med. 2004; 7: 136-139.

14. Novati S, Sacchi L, Chichino G, Scaglia M. Miasi foruncolosa da Cordylobia anthropophaga: descrizione di un caso proveniente dalla Tanzania. Parassitologia 1994; 36: 265-267. 
15. Pampiglione S, Canestri Trotti G. Miasi umana nasofaringea con reperto di larve di Oestrus ovis L. di secondo stadio. Biologia Oggi (1991); VI (4): 167-170.

16. Pampiglione S. Le miasi oculari dell'uomo in Italia: revisione critica dei casi descritti. Nuovi Ann. d'Ig. e Microbiol. 1957; Vol. VIII (4): 411-421.

17. Pampiglione S. La miasi da "Oestrus ovis" nell'uomo in Italia, malattia dei pastori. Attual. Med. 1958; 5: 1-4.

18. Pampiglione S, Trentini M, Marini M, Nunzi E, Rivasi F. Miasi umana da Dermatobia hominis: revisione della casistica italiana. Parassitologia 1998; 40 (Suppl. 1): 125.

19. Pampiglione S, Canestri Trotti G. Guida allo Studio della Parassitologia. Società Editrice Esculapio, Bologna, 1999, 304-317.

20. Sacchi L. Miasi, in: Scaglia M., Gatti S., Rondanelli E. G. Parassiti e parassitosi umane. Selecta Medica, Pavia, 2005, 627-630.

21. Scala A, Paz-Silva A, Suarez JL, et al. Chronobiology of Oestrus ovis (Diptera: Oestridae) in Sardinia, Italy: guidelines to chemoprophylaxis. J. Med. Entomol. 2002; 39: 652-657.

22. Suarez VH, Busetii MR, Mirando AO, Prévot F, Jacquiet P. Epidemiology of Oestrus ovis of sheep in Argentina's Western Pampas. Parsite 2004; 11: $405-$ 410.

23. Suarez JL, Scala A, Romero JA, et al. Analysis of the humoral immune response to Oestrus ovis in ovine. Vet. Parasitol. 2005; 134: 153-158.

24. Urbani C, Simonacci M, Castelli P, Maroli M. Miasi da Cordylobia antropophaga (Diptera: Calliphoridae): descrizione di un caso clinico e rassegna della letteratura. Parassitologia 1998; 40: 317-319.

25. Zardi EM, Iori A, Picardi A, Costantino S, Petrarca V. Myasis of a perineal fistola. Parassitologia 2002; 44: 201-201.

\section{Daniele Crotti}

Strada Comunale per Pilonico Paterno 4 06080 Pianello, Perugia

Tel.: +39075602372

E-mail:nenedc@tin.it 